\title{
Unoriented topological quantum field theory and link homology
}

\author{
VLADIMIR TURAEV \\ PAUL TURNER
}

\begin{abstract}
We investigate link homology theories for stable equivalence classes of link diagrams on orientable surfaces. We apply $(1+1)$-dimensional unoriented topological quantum field theories to Bar-Natan's geometric formalism to define new theories for stable equivalence classes.
\end{abstract}

57M25, 57R56; 81T40

\section{Introduction}

In this paper we consider link diagrams on orientable surfaces up to the relation of stable equivalence, that is up to homeomorphisms of surfaces, Reidemeister moves and the addition or subtraction of handles disjoint from the diagram. Stable equivalence classes of link diagrams have an equivalent formulation in terms of so called "virtual" link diagrams pioneered by Kauffman (see for example the review articles Kauffman and Manturov [8] and Fenn, Kauffman and Manturov [6] and references therein). Many constructions for link diagrams on $\mathbb{R}^{2}$ can be reproduced for stable equivalence classes of link diagrams on surfaces, for example, one can define the Jones polynomial.

In his seminal work [9] Khovanov provided a new way to look at the Jones polynomial of links in $\mathbb{R}^{3}$ interpreting it as the Euler characteristic of a homology theory. This approach provides an invariant that is not only stronger than the Jones polynomial but also has nice functorial properties with respect to link cobordisms. The Jones polynomial may be viewed as a state sum over the $2^{n}$ "smoothings" of an $n$-crossing link diagram on $\mathbb{R}^{2}$, with each state making a contribution to the polynomial. Each smoothing is a collection of circles being the result of resolving each crossing in one of two possible ways (indicated in Figure 3). Khovanov's insight was to associate a graded vector space to each smoothing and arrange these so that a certain topological quantum field theory can be used to define a differential leading to a chain complex associated to the link diagram. Remarkably the homotopy type of this complex is independent of the chosen diagram so its homology is an invariant. Bar-Natan has written a wonderful 
exposition of all in [4]. The functorial properties of this construction were conjectured by Khovanov [9], proven by Jacobsson [7] and later reproved in more generality by Bar-Natan [5].

Khovanov's work does not immediately extend to link diagrams on arbitrary orientable surfaces. One difficulty arising is the following. For classical links Khovanov's complex is constructed by organising the $2^{n}$ smoothings into a "cube" with each edge making a contribution to the differential. These edges join smoothings with different numbers of circles and the corresponding term of the differential may be seen as fusing two circles into one circle or splitting one circle into two. For links on surfaces this is no longer the case, and there may be edges joining smoothings with the same number of circles. This arises when the surface is part of the structure (as in the work of by Asaeda, Przytycki and Sikora [2]) and also for stable equivalence classes. In the latter case Manturov [12] solves this problem over the two element field $\mathbb{F}_{2}$ by setting the differential corresponding to such cube edges to be zero. The motivation for the current work was to find other possibilities for stable equivalence classes.

Bar-Natan's geometric complex [5] which is locally defined can be extended to diagrams on surfaces, but at the price of admitting nonorientable cobordisms. In this paper the key new idea is to define the notion of unoriented topological quantum field theory, which can then be applied to the geometric picture to obtain a link homology theory for stable equivalence classes of diagrams on surfaces.

Here is an outline of the paper. In Section 2 we define the notion of unoriented topological quantum field theory and study the underlying algebraic structure which includes a Frobenius algebra with additional structure. In dimension $1+1$ we classify isomorphism classes of unoriented TQFTs in terms of these Frobenius algebras. We include analysis of the particular case of rank-two theories, which will be used later to give link homologies. In Section 3 we follow Bar-Natan in defining a complex of cobordisms $[[D]]$ associated to a link diagram $D$ on a closed orientable surface. By introducing Bar-Natan's sphere, torus and 4-Tu relations we obtain an invariant of the link diagram on the surface. In Section 4 we apply an unoriented topological quantum field theory to the complex of cobordisms defined in Section 3 to obtain a complex of modules. Taking homology yields a link homology. Our main theorem is the following.

Theorem 4.4 Let $R$ be a commutative ring with unit. Let $\lambda, \mu, \beta, a, t \in R$ such that $a$ is invertible and the following relations are satisfied:

$$
\begin{gathered}
\mu \beta=\lambda \beta=0, \\
2 a \lambda \mu-a^{2} \mu^{2} \lambda^{2}-a^{2} \mu^{4} t=2 .
\end{gathered}
$$


Then the 5-tuple ( $\lambda, \mu, \beta, a, t)$ defines (the isomorphism class of) a link homology theory for stable equivalence classes of diagrams on orientable surfaces.

We end the paper by discussing a few examples.

\section{Unoriented topological quantum field theories}

In this section we define the notion of unoriented topological quantum field theory (briefly, TQFT). In the 1+1-dimensional case there is a classification in terms of Frobenius algebras with additional structure.

All the constructions can be formulated in the smooth and PL categories but we prefer to use the language of topological manifolds. Thus, by a manifold we shall mean a topological manifold. By a cobordism, we mean a triple $\left(W, M_{0}, M_{1}\right)$ where $W$ is a compact manifold whose boundary is a disjoint union of two closed manifolds $M_{0}, M_{1}$ (possibly void). A homeomorphism of cobordisms $\left(W, M_{0}, M_{1}\right) \rightarrow\left(W^{\prime}, M_{0}^{\prime}, M_{1}^{\prime}\right)$ is a homeomorphism $W \rightarrow W^{\prime}$ sending $M_{0}$ homeomorphically to $M_{0}^{\prime}$ and $M_{1}$ homeomorphically to $M_{1}^{\prime}$.

\subsection{The definition}

Fix an integer $d \geq 0$ and a commutative ring with unity $R$. We define in this subsection the notion of $(d+1)$-dimensional unoriented topological quantum field theory. Such a TQFT takes values in the category of projective $R$-modules of finite type (direct summands of $R^{n}$ with $n=0,1, \ldots$ ) and $R$-linear homomorphisms. In the case where $R$ is a field, projective $R$-modules of finite type are just finite-dimensional vector spaces over $R$.

Definition 2.1 A $(d+1)$-dimensional unoriented TQFT $(A, \tau)$ assigns a projective $R$-module of finite type $A_{M}$ to any closed $d$-dimensional manifold $M$, an $R$-isomorphism $f_{\#}: A_{M} \rightarrow A_{M^{\prime}}$ to any homeomorphism of $d$-dimensional manifolds $f: M \rightarrow M^{\prime}$, and an $R$-homomorphism $\tau(W): A_{M_{0}} \rightarrow A_{M_{1}}$ to any $(d+1)-$ dimensional cobordism $\left(W, M_{0}, M_{1}\right)$. These modules and homomorphisms should satisfy the following seven axioms.

(1) For any homeomorphisms of closed $d$-dimensional manifolds $f: M \rightarrow M^{\prime}$ and $f^{\prime}: M^{\prime} \rightarrow M^{\prime \prime}$, we have $\left(f^{\prime} f\right)_{\#}=f_{\#}^{\prime} f_{\#}$. The isomorphism $f_{\#}: A_{M} \rightarrow A_{M^{\prime}}$ is invariant under isotopies of $f$. 
(2) For any disjoint closed $d$-dimensional manifolds $M, N$, there is an isomorphism $A_{M \amalg N}=A_{M} \otimes A_{N}$, natural with respect to homeomorphisms, where $\otimes=\otimes_{R}$ is the tensor product over $R$.

(3) $A_{\varnothing}=R$ where the empty set is considered as a $d$-dimensional manifold (for any $d)$.

(4) The homomorphism $\tau$ associated with cobordisms is natural with respect to homeomorphisms of cobordisms.

(5) If a $(d+1)$-dimensional cobordism $W$ is a disjoint union of cobordisms $W_{1}, W_{2}$, then under the identifications in axiom $(2), \tau(W)=\tau\left(W_{1}\right) \otimes \tau\left(W_{2}\right)$.

(6) For a cobordism $\left(W, M_{0}, M_{1}\right)$ obtained from $(d+1)$-dimensional cobordisms $\left(W_{0}, M_{0}, N\right)$ and $\left(W_{1}, N^{\prime}, M_{1}\right)$ by gluing along a homeomorphism $f: N \rightarrow N^{\prime}$,

$$
\tau(W)=\tau\left(W_{1}\right) \circ f_{\#} \circ \tau\left(W_{0}\right): A_{M_{0}} \rightarrow A_{M_{1}} .
$$

(7) For any closed $d$-dimensional manifold $M$, we have

$$
\tau(M \times[0,1], M \times 0, M \times 1)=\mathrm{Id}: A_{M} \rightarrow A_{M}
$$

where we identify $M \times 0$ and $M \times 1$ with $M$ in the obvious way.

The pair $(A, \tau)$ is isomorphic to another $\left(A^{\prime}, \tau^{\prime}\right)$ if for each closed $d$-manifold $M$ there is an isomorphism $\eta_{M}: A_{M} \rightarrow A_{M}^{\prime}$, natural with respect to homeomorphisms and cobordisms, multiplicative with respect to disjoint union and such that $\eta_{\varnothing}=\operatorname{Id}_{R}$.

This definition is essentially the one of Atiyah [3] but all references to orientations of manifolds are suppressed. The axioms (1)-(7) constitute a special case of a detailed axiomatic definition of TQFT's given in Turaev [13], Chapter III in a framework of so-called space-structures. For more on the naturality in axioms 2 and 4, the reader is referred to [13, p. 121]; otherwise a knowledge of [13] will not be required and the language of space-structures will not be used.

In dimension $1+1$ a related idea has already appeared in Alexeevski and Natanzon [1] in the context of open-closed field theory. The definition presented there of a Klein topological field theory is, however, rather different from that above, justifying a separate treatment here.

Note that the tensor product in axiom (2) is unordered so that the modules $A_{M} \otimes A_{N}$ and $A_{N} \otimes A_{M}$ are the same and not merely isomorphic. The unordered tensor product of a finite family of modules is obtained by considering all possible orderings of this family, forming the corresponding ordered tensor products and then identifying the 
resulting modules along the obvious isomorphisms induced by permutations. We have to use here the unordered tensor product since we want to apply axiom (2) to nonconnected $d$-manifolds without fixing any order in the set of connected components.

\subsection{Extended Frobenius algebras}

We recall that a commutative Frobenius algebra over $R$ is a unital, commutative $R-$ algebra $V$ which as an $R$-module is projective of finite type, together with a module homomorphism $\epsilon: V \rightarrow R$ such that the bilinear form $\langle-,-\rangle: V \otimes V \rightarrow R$ defined by $\langle v, w\rangle=\epsilon(v w)$ is nondegenerate, ie, the adjoint homomorphism $V \rightarrow V^{*}$ is an isomorphism. The homomorphism $\epsilon$ is called the co-unit and it is useful to define a coproduct $\Delta: V \rightarrow V \otimes V$ by $\Delta(v)=\sum_{i} v_{i}^{\prime} \otimes v_{i}^{\prime \prime}$ being the unique element such that for all $w \in V, v w=\sum_{i} v_{i}^{\prime}\left\langle v_{i}^{\prime \prime}, w\right\rangle$.

The map $\Delta: V \rightarrow V \otimes V$ satisfies

$$
(\mathrm{Id} \otimes m) \circ(\Delta \otimes \mathrm{Id})=\Delta \circ m=(m \otimes \mathrm{Id}) \circ(\mathrm{Id} \otimes \Delta)
$$

and

$$
(\mathrm{Id} \otimes \epsilon) \circ \Delta=\mathrm{Id}=(\epsilon \otimes \mathrm{Id}) \circ \Delta
$$

where $m: V \otimes V \rightarrow V$ is the multiplication in $V$ and $i: R \rightarrow V$ is the unit map.

An unoriented $(d+1)$-dimensional TQFT $(A, \tau)$ has an underlying Frobenius algebra equipped with an involution of Frobenius algebras as we now discuss.

For the underlying $R$-module we take $V=A_{S^{d}}$, where $S^{d}$ is the standard unit sphere in Euclidean space $\mathbb{R}^{d+1}$. Note that any other sphere $S$ in $\mathbb{R}^{d+1}$ can be related to the standard sphere $S^{d}$ by parallel translations and homotheties which allows us to canonically identify the corresponding vector spaces (courtesy of axiom (1) in Definition 2.1).

The unit $i$ and the co-unit $\epsilon$ come from the $(d+1)$-dimensional unit ball $B$ in $\mathbb{R}^{d+1}$ viewed as a cobordism from the empty set to $S^{d}$ and as a cobordism from $S^{d}$ to the empty set respectively.

The product is obtained by considering a ball $W_{00}$ of radius three in $\mathbb{R}^{d+1}$ with two interior balls of radius 1 removed. This can be viewed as a cobordism from the two internal spheres to the external sphere which, using the canonical identifications above, gives a map $m: V \otimes V \rightarrow V$. Although not necessary as part of the definition we note that the coproduct has geometric interpretation by viewing $W_{00}$ as a cobordism from the sphere of radius three to the two internal spheres.

The involution $\phi: V \rightarrow V$ is induced by an orientation-reversing homeomorphism $\chi: S^{d} \rightarrow S^{d}$. They are all isotopic so $\phi$ is well defined. 
Proposition 2.2 The $R$-module $V$ equipped with structure maps $m, i, \epsilon$ defined above is a Frobenius algebra and $\phi$ is an involution of Frobenius algebras.

Proof It follows from the familiar oriented case that $(V, m, i, \epsilon)$ is a Frobenius algebra. We observe that $\phi^{2}=$ Id since $\chi \circ \chi$ is isotopic to the identity on $S^{d}$.

Next we claim that $\phi$ is a homomorphism of Frobenius algebras. Note that an orientation-reversing homeomorphism $S^{d} \rightarrow S^{d}$ extends to $B$, hence by axiom (4) in Definition 2.1, $\phi$ preserves the unit and counit. There is also an orientation-reversing homeomorphism $W_{00} \rightarrow W_{00}$ mapping each boundary component to itself showing that $\phi \circ m \circ(\phi \otimes \phi)=m$, and hence since $\phi^{2}=\mathrm{Id}$, we see $m \circ(\phi \otimes \phi)=\phi \circ m$

One further piece of structure can be identified in the form of an element $\theta \in V$. Consider the punctured projective space $\mathbb{P}$ of dimension $d+1$ viewed as a cobordism between the empty set and $\partial \mathbb{P}$. Now we identify $\partial \mathbb{P}$ with $S^{d}$ via a map $f: \partial \mathbb{P} \rightarrow S^{d}$ and set $\theta=f_{\#} \circ \tau(\mathbb{P})(1)$. There is an involution $T$ on $\mathbb{P}$ given by the negation of one coordinate which by axiom (4) in Definition 2.1 gives $T_{\#}(\tau(\mathbb{P})(1))=\tau(\mathbb{P})(1)$. Now $T$ reverses the orientation of the boundary and thus if $f^{\prime}$ is another homeomorphism $\partial \mathrm{P} \rightarrow S^{d}$ nonisotopic to $f$ then $f^{\prime} \circ T$ is isotopic to $f$ and we have

$$
f_{\#}^{\prime}(\tau(\mathbb{P})(1))=f_{\#}^{\prime}\left(T_{\#}(\tau(\mathbb{P})(1))\right)=f_{\#}(\tau(\mathbb{P})(1)) .
$$

This shows that $\theta$ is well defined.

Lemma 2.3 $\phi(\theta)=\theta$.

Proof The map $T: \mathbb{P} \rightarrow \mathbb{P}$ defined above reverses the orientation on $\partial \mathbb{P}$ so by axiom (4) in Definition 2.1 we have $\phi(\theta)=\theta$.

When $d$ is odd there is a stronger relation between $\phi$ and $\theta$.

Proposition 2.4 If $d$ is odd then $\phi(\theta v)=\theta v$ for all $v \in V$.

Proof Consider two disjoint balls $B_{1}$ and $B_{2}$ in $\mathbb{P}^{d+1}$ in the complement of $\mathbb{P}^{d}$ in $\mathbb{P}^{d+1}$. Let $S_{i}=\partial B_{i}, i=1,2$ and consider the cobordism $\left(W, S_{1}, S_{2}\right)$ where $W=\mathbb{P}^{d+1}-\operatorname{Int}\left(B_{1} \sqcup B_{2}\right)$. It is clear that $W$ is a connected sum $C \# \mathbb{P}^{d+1}$ where $C$ is homeomorphic to $S^{d} \times I$. As the $d$-sphere separating $W$ into two connected summands we take the boundary of a regular neighbourhood of $\mathbb{P}^{d}$ in $\mathbb{P}^{d+1}$. Now choose an arbitrary orientation of $S_{1}$ and endow $S_{2}$ with the orientation such that $\tau(C): V \cong A_{S_{1}} \rightarrow A_{S_{2}} \cong V$ is the identity homomorphism. We can then use the decomposition of $W$ to compute $\tau(W)(v)=\theta v$. 
Since $d$ is odd, and hence $\mathbb{P}^{d+1}$ unorientable, there is a homeomorphism $T: W \rightarrow W$ which reverses the orientation of $S_{2}$ while leaving the orientation of $S_{1}$ unchanged. This is obtained by moving $B_{2}$ by an isotopy in $\mathbb{p}^{d+1}$ back to itself passing through $\mathbb{P}^{d}$ exactly once. Since $T$ reverses the orientation of $S_{2}$, using the identifications above we have $\left.T\right|_{S_{2}}=\phi: V \rightarrow V$. On $S_{1}$ we have $\left.T\right|_{S_{1}}=$ Id: $V \rightarrow V$. By axiom (4) in Definition 2.1 the following diagram commutes.

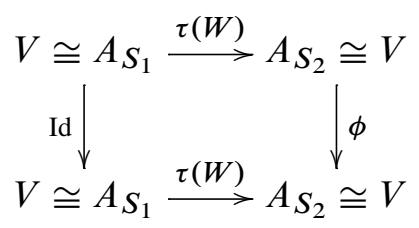

Chasing around this diagram gives $\phi(\theta v)=\theta v$ as required.

In fact in dimension $1+1$ the assignment of the structure above induces a bijection from isomorphism classes of TQFTs to a special class of Frobenius algebras.

Definition 2.5 An extended Frobenius algebra is a Frobenius algebra $(V, m, i, \epsilon)$ together with an involution of Frobenius algebras $\phi: V \rightarrow V$ and an element $\theta \in V$ satisfying the following two axioms.

(1) $\phi(\theta v)=\theta v$, for all $v \in V$.

(2) $m(\phi \otimes \operatorname{Id})(\Delta(1))=\theta^{2}$.

Two extended Frobenius algebras $(V, \theta, \phi)$ and $\left(V^{\prime}, \theta^{\prime}, \phi^{\prime}\right)$ are isomorphic if there exists an isomorphism of Frobenius algebras $g: V \rightarrow V^{\prime}$ such that $g(\theta)=\theta^{\prime}$ and $g \circ \phi=\phi^{\prime} \circ g$.

We note that in [1] such algebras appear as "structure algebras" in which the part coming from open boundaries is trivial.

Example 2.6 Let $N$ be odd and let $V=\mathbb{R}[x] / x^{N}$. Taking $\phi=\operatorname{Id}$ and $\theta=\sqrt{N} x^{\frac{N-1}{2}}$ we see that $(V, \phi, \theta)$ is an extended Frobenius algebra. In this case $\phi$ is obviously an involution of Frobenius algebras satisfying axiom (1). Axiom (2) is reduced to $m(\Delta(1))=\theta^{2}$ which holds for our choice of $\theta$ since $m(\Delta(1))=N x^{N-1}$.

Example 2.7 Let $V$ be the two dimensional vector space over $\mathbb{F}_{2}$ on generators 1 and $x$. This may be given the structure of Frobenius algebra by defining $i(1)=1$, $x^{2}=x, \epsilon(1)=0, \epsilon(x)=1$. Taking $\theta=0$ and $\phi(1)=1$, then $\phi(x)=1+x$ turns this into an extended Frobenius algebra. 
There are some useful elementary consequences of the definition.

Lemma 2.8 (i) $m(\phi \otimes \operatorname{Id})(\Delta(v))=m(\phi \otimes \operatorname{Id})(\Delta(1)) v$ for all $v \in V$.

(ii) $m(\phi \otimes \mathrm{Id})(\Delta(v))=\theta^{2} v$ for all $v \in V$.

(iii) $m(\Delta(\theta))=\theta^{3}$.

Proof To prove (i) it suffices to notice that $\Delta(v)=(v \otimes 1) \Delta(1)$. Part (ii) follows immediately from (i). For (iii) we have

$$
m(\Delta(\theta))=m((\theta \otimes 1) \Delta(1))=m(\phi \otimes 1)((\theta \otimes 1) \Delta(1))=m(\phi \otimes 1)(\Delta(\theta))=\theta^{3} . \square
$$

Proposition 2.9 Isomorphism classes of unoriented 1+1-dimensional TQFTs over $R$ are in bijective correspondence with isomorphism classes of extended Frobenius algebras over $R$.

Proof We have already shown that an unoriented 1+1-dimensional TQFT has an underlying Frobenius algebra over $R$ and comes equipped with an involution of Frobenius algebras $\phi$. Furthermore we have defined the element $\theta$ and shown in Proposition 2.4 that $\phi(\theta v)=\theta v$ for all $v \in V$. It remains to show axiom (2) in the definition of extended Frobenius algebra. To see this we glue the ends of an oriented punctured cylinder together in a nonorientation-preserving way, regarding the result $W$ as a cobordism $(W, \varnothing, \partial W)$. We can use the original orientation of the cylinder to orient $\partial W$ and hence identify $A_{\partial W}$ with $V$. One can easily compute $\tau(W): R \rightarrow V$ to be the map taking 1 to $m(\phi \otimes \operatorname{Id})(\Delta(1))$. On the other hand $W$ is a punctured Klein bottle and hence homeomorphic to the punctured connected sum of two projective planes. Thus $\tau(W)(1)=\theta^{2}$. It is clear that the isomorphism class of this algebra depends only on the isomorphism class of the TQFT.

We now show that every extended Frobenius algebra $(V, \theta, \phi)$ gives rise to an unoriented TQFT. Given a connected closed 1-manifold $M$ we define the set $A_{M}$ by

$$
A_{M}=\left\{(\gamma, v) \mid \gamma: S^{1} \rightarrow M \text { a homeomorphism, } v \in V\right\} / \sim
$$

where

$$
\begin{aligned}
(\gamma, v) \sim\left(\gamma^{\prime}, v^{\prime}\right) \text { iff either } \gamma \text { is isotopic to } \gamma^{\prime} \text { and } v & =v^{\prime} \\
\text { or } \gamma \text { is not isotopic to } \gamma^{\prime} \text { and } v & =\phi\left(v^{\prime}\right) .
\end{aligned}
$$

Note that there are two isotopy classes of homeomorphisms $S^{1} \rightarrow M$. Now pick any homeomorphism $h: S^{1} \rightarrow M$ and define a map (of sets) $\tilde{h}: A_{M} \rightarrow V$ by

$$
\tilde{h}(\gamma, v)= \begin{cases}v & \text { if } \gamma \text { is isotopic to } h \\ \phi(v) & \text { else. }\end{cases}
$$


This map is a bijection and moreover, since $V$ is an $R$-module, we can use it to turn $A_{M}$ into an $R$-module. It is easy to check that the $R$-module structure is independent of the isotopy class of $h$. Thus to a closed 1-manifold $M$ we have assigned an $R$-module $A_{M}$. If $M$ is not connected we simply write $M=M_{1} \sqcup \cdots \sqcup M_{k}$ and let $A_{M}=\bigotimes A_{M_{i}}$. We define $A_{\varnothing}=R$.

Now let $f: M \rightarrow M^{\prime}$ be a homeomorphism of connected 1-manifolds (up to isotopy there are two choices). Define $f_{\#}: A_{M} \rightarrow A_{M^{\prime}}$ by $f_{\#}(\gamma, v)=(f \circ \gamma, v)$. One can check this is a well defined isomorphism of $R$-modules. This extends to homeomorphisms of nonconnected manifolds by multiplicativity.

We now wish to define $\tau(W): A_{M_{0}} \rightarrow A_{M_{1}}$ for a cobordism $\left(W, M_{0}, M_{1}\right)$. We separate the cases of orientable and nonorientable surfaces.

Suppose first that $W$ is orientable and connected. Choose an orientation for $W$ and decompose it into basic pieces (caps, cups and pairs of pants). We can appeal to the usual case of oriented TQFT to define $\tau(W)$ which is independent of the decomposition. We claim that $\tau(W)$ is independent of the orientation chosen for $W$. Indeed by choosing the opposite orientation, each piece in the decomposition also has the opposite orientation. Consider, for example a pair of pants $(P, M, N)$ occurring in the decomposition and consider the following diagram where the top route corresponds to one orientation and the bottom route to the other.

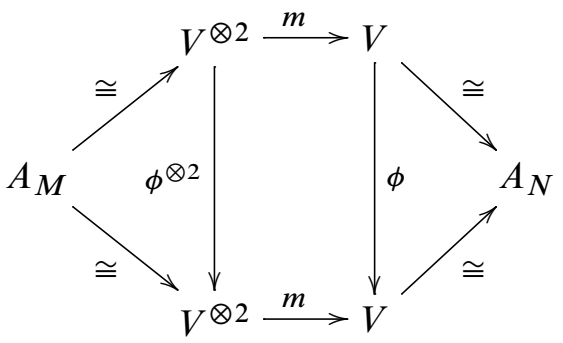

Note that if a connected $1-$ manifold $M$ is oriented then there is a canonical identification of $A_{M}$ with $V$, using the map $\tilde{h}: A_{M} \rightarrow V$ defined by an orientation-preserving homeomorphism $h: S^{1} \rightarrow M$ (here $S^{1}$ is given the anticlockwise orientation). By reversing the orientation of $M$ the identification is given by $\phi \circ \widetilde{h}$. Thus the left and right triangles in the diagram above commute. Since $\phi$ is a map of Frobenius algebras the middle square also commutes, hence the two routes give the same map. Similar arguments hold for the other basic surfaces, showing that $\tau(W)$ is independent of the orientation. Furthermore, the properties of oriented TQFTs guarantee that $\tau(W)$ is natural with respect to homeomorphisms. For nonconnected $W$ we extend the above multiplicatively. 
Suppose now that $W$ is nonorientable and connected. We may present $W$ as a connected sum of an orientable surface $W^{\text {or }}$ and $n$ projective planes, $W=W^{\text {or }} \# n \mathbb{R} P^{2}$. Note that $\partial W^{\text {or }}=\partial W$ and that the homomorphism $\tau\left(W^{\text {or }}\right): A_{M_{0}} \rightarrow A_{M_{1}}$ is defined by the orientable case above. Now choose an identification $A_{M_{1}} \cong V^{\otimes k}$ and define $\psi_{n}: A_{M_{1}} \cong V^{\otimes k} \rightarrow V^{\otimes k} \cong A_{M_{1}}$ to be the identity on all factors except one where it is multiplication by $\theta^{n}$. We define $\tau(W)=\psi_{n} \circ \tau\left(W^{\text {or }}\right)$.

A priori this depends on the identification $A_{M_{1}} \cong V^{\otimes k}$, the choice of factor in $\psi_{n}$ and the decomposition $W=W^{\text {or }} \# n \mathbb{R} P^{2}$. It follows from the properties of $V$ that the factor in $\psi_{n}$ does not matter. Moreover, since $\phi(\theta)=\theta$ the definition is independent of the identification $A_{M_{1}} \cong V^{\otimes k}$. Now suppose that we decompose $W$ differently as $W=\bar{W}^{\text {or }} \# n \mathbb{R} P^{2}$ then there is a homeomorphism $g: \bar{W}^{\text {or }} \rightarrow W^{\text {or }}$ taking each boundary component to itself. On each such boundary component $g$ is either isotopic to the identity or is an orientation-reversing homeomorphism. By the naturality of $\tau$ for orientable surfaces we have the following commutative diagram.

$$
\begin{gathered}
A_{M_{0}} \stackrel{\tau\left(W^{\circ r}\right)}{\longrightarrow} A_{M_{1}} \\
\left(\left.g\right|_{M_{0}}\right)_{\#} \downarrow \\
A_{M_{0}} \stackrel{\tau\left(\bar{W}^{\text {or }}\right)}{\longrightarrow} A_{M_{1}}^{\downarrow}\left(\left.g\right|_{\left.M_{1}\right)_{\#}}\right.
\end{gathered}
$$

We also have the following commutative diagram.

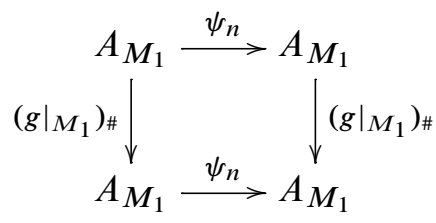

Commutativity is immediate for a component of $M_{1}$ on which $\left.g\right|_{M_{1}}$ is isotopic to the identity and follows from the relation $\phi(\theta v)=\theta v$ on a component where $\left.g\right|_{M_{1}}$ is an orientation-reversing homeomorphism. Combining these two diagrams shows that $\tau(W)$ is independent of the decomposition above. We may also choose a different number of projective planes in the decomposition, replacing any three by a torus and a projective plane. However, in this case we may write $W=W^{\text {or }} \# T^{2} \#(n-2) \mathbb{R} P^{2}$ and $\tau(W)$ can be computed as $\tau\left(W^{\text {or }}\right)$ multiplied on one factor by $m(\Delta(1)) \theta^{n-2}$. However, by Lemma 2.8 we have $m(\Delta(1)) \theta^{n-2}=m(\Delta(\theta)) \theta^{n-3}=\theta^{n}$ showing that $\tau(W)$ is again independent of the decomposition. For a nonconnected cobordism we extend multiplicatively.

What remains is to show that the structure defined above verifies axioms (1)-(7) in Definition 2.1. These are all easy to show with the exception of the gluing axiom (6). 
We leave the others as an exercise and focus on proving this axiom, for which the following definition is useful.

Definition 2.10 A cobordism $\left(W_{0}, M_{0}, N\right)$ is nice if for all $\left(W_{1}, N^{\prime}, M_{1}\right)$ and homeomorphisms $f: N \rightarrow N^{\prime}$ we have

$$
\tau(W)=\tau\left(W_{1}\right) \circ f_{\#} \circ \tau\left(W_{0}\right)
$$

where $\left(W, M_{0}, M_{1}\right)$ is the result of gluing $W_{0}$ to $W_{1}$ along $f$.

It is easy to check the following lemma.

Lemma 2.11 (i) If the cobordism $\left(W_{0}, M_{0}, N_{0}\right)$ is obtained from $\left(W_{0}^{\prime}, M_{0}^{\prime}, N_{0}^{\prime}\right)$ and $\left(W_{0}^{\prime \prime}, M_{0}^{\prime \prime}, N_{0}^{\prime \prime}\right)$ by gluing along a homeomorphism $g: N_{0}^{\prime} \rightarrow M_{0}^{\prime \prime}$, and $W_{0}^{\prime}$ and $W_{0}^{\prime \prime}$ are nice, then $W_{0}$ is also nice.

(ii) If $\left(W_{0}, M_{0}, N_{0}\right)$ and $\left(W_{1}, M_{1}, N_{1}\right)$ are oriented and $f: N_{0} \rightarrow M_{1}$ is orientation preserving then $\tau(W)=\tau\left(W_{1}\right) \circ f_{\#} \circ \tau\left(W_{0}\right)$.

Any surface may be decomposed into a composition of cobordisms of the six basic types (up to ordering) indicated in Figure 1 where the input boundary is always at the top (the picture on the right at the bottom represents a twice punctured projective plane).
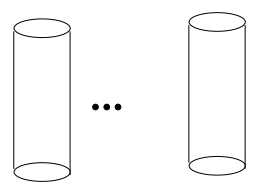

(1)

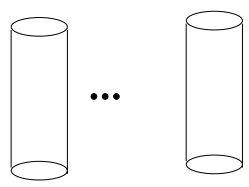

(4)

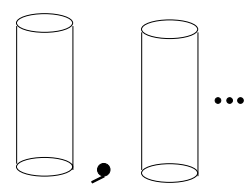

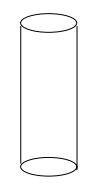

(2)

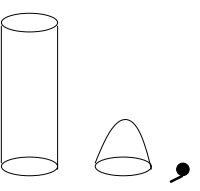

(5)
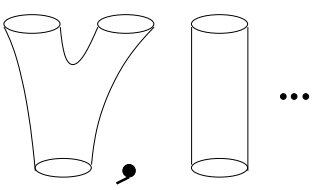

(3)
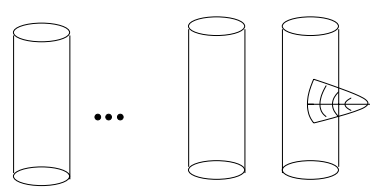

(6)

Figure 1: Basic cobordism types

In order to prove that the gluing axiom holds we will show that any cobordism of the six types in Figure 1 is nice. Thus by Lemma 2.11 (i) we will have the desired result. 
First we claim that any cobordism $W_{0}$ of type (2) is nice. To see this let $\left(W_{1}, N^{\prime}, M_{1}\right)$ be a cobordism which we glue to $W_{0}$ along a homeomorphism $f: N \rightarrow N^{\prime}$. We can present $W_{1}$ as $W_{1}=W_{1}^{\text {or }} \# n \mathbb{R} P^{2}(n \geq 0)$. We now orient $W_{1}^{\text {or }}$ and choose orientations for each of the cylinders and the pair of pants in $W_{0}$ such that $f$ is orientation preserving. Let $W_{2}$ be the result of gluing $W_{1}^{\text {or }}$ to $W_{0}$ along $f$ so that $W=W_{2} \# n \mathbb{R} P^{2}$. By Lemma 2.11 (ii) we have $\tau\left(W_{2}\right)=\tau\left(W_{1}^{\text {or }}\right) \circ f_{\#} \circ \tau\left(W_{0}\right)$. Thus

$$
\begin{aligned}
\tau(W) & =\psi_{n} \circ \tau\left(W_{2}\right)=\psi_{n} \circ\left(\tau\left(W_{1}^{\text {or }}\right) \circ f_{\#} \circ \tau\left(W_{0}\right)\right) \\
& =\left(\psi_{n} \circ \tau\left(W_{1}^{\text {or }}\right)\right) \circ f_{\#} \circ \tau\left(W_{0}\right)=\tau\left(W_{1}\right) \circ f_{\#} \circ \tau\left(W_{0}\right) .
\end{aligned}
$$

Similar arguments show that cobordisms of type (1), (4) and (5) are nice.

For a cobordism of type (6) we decompose $W_{0}$ and $W_{1}$ as $W_{0}=W_{0}^{\text {or }} \# \mathbb{R} P^{2}$ and $W_{1}=W_{1}^{\text {or }} \# n \mathbb{R} P^{2}$. Now let $W_{2}$ be the result of gluing $W_{1}^{\text {or }}$ to $W_{0}^{\text {or }}$ along $f$. We can write $W=W_{2} \#(n+1) \mathbb{R} P^{2}$. Thus

$$
\begin{aligned}
\tau(W) & =\psi_{n+1} \circ \tau\left(W_{2}\right)=\psi_{n+1} \circ\left(\tau\left(W_{1}^{\text {or }}\right) \circ f_{\#} \circ \tau\left(W_{0}^{\text {or }}\right)\right) \\
& =\left(\psi_{1} \circ \tau\left(W_{1}^{\text {or }}\right)\right) \circ f_{\#} \circ\left(\psi_{n} \circ \tau\left(W_{0}^{\text {or }}\right)\right)=\tau\left(W_{1}\right) \circ f_{\#} \circ \tau\left(W_{0}\right) .
\end{aligned}
$$

The most difficult case is for cobordisms of type (3). We decompose $W_{1}$ into the connect sum $W_{1}^{\text {or }} \# n \mathbb{R} P^{2}$ and we orient $W_{1}^{\text {or }}$ in an arbitrary way. If there is an orientation of $W_{0}$ such that $f$ is an orientation-preserving homeomorphism then we are done by the same arguments as above. If there is no such orientation we decompose $W_{1}$ into the composition of $W_{2}$ and $W_{3}$ glued along a (canonical) map $g$ where $W_{2}$ is of type (2). Since by the above $W_{2}$ is nice we have $\tau\left(W_{1}\right)=\tau\left(W_{3}\right) \circ g_{\#} \circ \tau\left(W_{2}\right)$. Now let $W_{4}$ be the result of gluing $W_{2}$ to $W_{0}$ along $f$, which results in a number of cylinders and a twice punctured Klein bottle. We can compute $\tau\left(W_{4}\right)=\psi_{2} \circ \tau\left(W_{4}^{\text {or }}\right)$. On the other hand by using Lemma 2.8 (ii) we have $\tau\left(W_{4}\right)=\tau\left(W_{2}\right) \circ f_{\#} \circ \tau\left(W_{0}\right)$. Finally we note that $W$ is the result of gluing $W_{3}$ to $W_{4}$ along $g$ and so

$$
\begin{aligned}
\tau(W) & =\psi_{n+2} \circ \tau\left(W^{\text {or }}\right)=\psi_{n+2} \circ\left(\tau\left(W_{3}^{\text {or }}\right) \circ g_{\#} \circ \tau\left(W_{4}^{\text {or }}\right)\right) \\
& =\left(\psi_{n} \circ \tau\left(W_{3}^{\text {or }}\right)\right) \circ g_{\#} \circ\left(\psi_{2} \circ \tau\left(W_{4}^{\text {or }}\right)\right)=\tau\left(W_{3}\right) \circ g_{\#} \circ \tau\left(W_{4}\right) \\
& =\tau\left(W_{3}\right) \circ g_{\#} \circ \tau\left(W_{2}\right) \circ f_{\#} \circ \tau\left(W_{0}\right)=\tau\left(W_{1}\right) \circ f_{\#} \circ \tau\left(W_{0}\right) .
\end{aligned}
$$

The gluing axiom now follows since all the pieces in the decomposition are nice.

Thus we have defined an unoriented TQFT. Standard arguments now show that the isomorphism class of this TQFT depends only on the isomorphism class of the extended Frobenius algebra. Moreover, the underlying extended Frobenius algebra is clearly $(V, \theta, \phi)$ so the construction provides the required inverse. 
We remark that in order to use the underlying Frobenius algebra to make computations we must choose for each closed 1-manifold $\Gamma$ an identification $\gamma: S^{1} \sqcup \cdots \sqcup S^{1} \rightarrow \Gamma$ which gives an isomorphism $\tau(\gamma): V^{\otimes r} \rightarrow A_{\Gamma}$ where $r$ is the number of components of $\Gamma$.

\subsection{Rank-two aspherical theories}

In this subsection we wish to study rank-two unoriented TQFTs satisfying the condition $\tau\left(S^{2}\right)=0$. For convenience we refer to these as rank-two aspherical unoriented TQFTs.

Lemma 2.12 Let $(A, \tau)$ be a rank-two aspherical unoriented TQFT and let $S$ be any sphere and $T$ any torus. Then $\tau(S)=0$ and $\tau(T)=2$.

Proof We have a standard torus constructed from discs and the surface $W_{00}$ (a pair of pants surface) which evaluates to 2 because the theory is rank-two. It follows from this, the fact that the TQFT is aspherical and axiom (4) in Definition 2.1 that any sphere evaluates to 0 and any torus evaluates to 2 .

The following is an immediate corollary to Proposition 2.9.

Proposition 2.13 The isomorphism classes of rank-two aspherical unoriented 1+1dimensional TQFTs over $R$ are in bijective correspondence with the isomorphism classes of rank-two extended Frobenius algebras over $R$ satisfying $\epsilon(i(1))=0$, where $\epsilon$ and $i$ are the counit and unit of the Frobenius algebra.

We now wish to classify the Frobenius algebras appearing in the previous proposition. Let $K=\mathbb{Z}[a, f, t, \lambda, \mu, \beta] / I$ where $I$ is the ideal generated by

$$
a f=1, \quad \mu \beta=\lambda \beta=0 \text { and } 2 a \lambda \mu-a^{2} \mu^{2} \lambda^{2}-a^{2} \mu^{4} t=2 .
$$

Now set $U=K\{1, x\}$ and define multiplication by

$$
11=1, \quad 1 x=x 1=x \quad \text { and } \quad x x=\left(\beta-a \lambda^{2}-a \mu^{2} t\right) x+t 1
$$

and comultiplication by

$$
\begin{gathered}
\Delta(1)=f(1 \otimes x+x \otimes 1)-\left(\beta-a \lambda^{2}-a \mu^{2} t\right) f 1 \otimes 1, \\
\Delta(x)=f x \otimes x+f t 1 \otimes 1 .
\end{gathered}
$$

Define a unit and counit by

$$
\epsilon(1)=0, \quad \epsilon(x)=a \quad \text { and } \quad i(1)=1 .
$$


Let

$$
\theta=\lambda 1+\mu x
$$

and define $\phi: U \rightarrow U$ by

$$
\phi(1)=1 \text { and } \phi(x)=\beta 1+x .
$$

Proposition 2.14 The triple $(U, \theta, \phi)$ defined above is an extended Frobenius algebra over $K$ satisfying $\epsilon(i(1))=0$.

Proof The proof is purely computational and hence omitted. The one thing worth pointing out is that the relations in $K$ imply $2 \beta=0$.

Given another ring with unity $R$ and a ring homomorphism $\psi: K \rightarrow R$ we can use $\psi$ to view $R$ as an $K$-module. We set $U_{\psi}=U \otimes_{K} R$ and define a comultiplication and counit by $\Delta \otimes 1$ and $\epsilon \otimes 1$. Since $U$ is a Frobenius algebra over $K$, it follows that $U_{\psi}$ is a Frobenius algebra over $R$. The resulting triple $\left(U_{\psi}, \theta \otimes 1, \phi \otimes \mathrm{Id}\right)$ is an extended Frobenius algebra over $R$ and it too satisfies $\epsilon(i(1))=0$.

The example in Proposition 2.14 is universal for rank-two theories in the sense of the following proposition.

Proposition 2.15 Let $R$ be a commutative ring with unit and let $\left(V, \theta^{\prime}, \phi^{\prime}\right)$ be a ranktwo extended Frobenius algebra over $R$ satisfying $\epsilon(i(1))=0$. Then there exists a ring homomorphism $K \rightarrow R$ such that $\left(U_{\psi}, \theta \otimes 1, \phi \otimes \mathrm{Id}\right)$ is isomorphic to $\left(V, \theta^{\prime}, \phi^{\prime}\right)$.

Proof Any rank-two Frobenius algebra $V$ over $R$ satisfying $\epsilon(i(1))=0$ is of the following form (see Khovanov [10]). As an $R$-module we can write $V=R\{1, x\}$ and there are elements $a, f, h, t \in R$ such that $a f=1$. In terms of these elements multiplication is defined by

$$
11=1, \quad 1 x=x 1=x \quad \text { and } \quad x x=h x+t 1
$$

and comultiplication is defined by

$$
\Delta(1)=f(1 \otimes x+x \otimes 1)-h f 1 \otimes 1 \quad \text { and } \quad \Delta(x)=f x \otimes x+f t 1 \otimes 1 .
$$

The unit and counit are given by

$$
\epsilon(1)=0, \quad \epsilon(x)=a \quad \text { and } \quad i(1)=1 .
$$

Suppose now $\theta \in V$ and $\phi: V \rightarrow V$ such that $(V, \theta, \phi)$ is an extended Frobenius algebra. Since $\phi$ is a map of Frobenius algebras we must have $\phi(1)=1$. Now write

$$
\theta=\lambda 1+\mu x \text { and } \phi(x)=\beta 1+\gamma x .
$$


We have $\epsilon(x)=\epsilon(\phi(x))$ from which we have

$$
a=\epsilon(x)=\epsilon(\beta 1+\gamma x)=\beta \epsilon(1)+\gamma \epsilon(x)=\gamma a \text {. }
$$

Multiplying by $f$ and recalling that $a f=1$ we see $\gamma=1$.

By axiom (1) of Definition 2.5 we have $\theta=\phi(\theta)$ and $\theta x=\phi(\theta x)$. These two relations imply that $\mu \beta=0$ and $\lambda \beta=0$.

By axiom (2) we have $m(\phi \otimes \mathrm{Id})\left((\Delta(1))=\theta^{2}\right.$. Now the left-hand side is equal to $(f \beta-h f) 1+2 f x$ and the right-hand side is equal to $\left(\lambda^{2}+\mu^{2} t\right) 1+\left(2 \lambda \mu+\mu^{2} h\right) x$. Thus we have

$$
\lambda^{2}+\mu^{2} t=f \beta-h f \quad \text { and } \quad 2 \lambda \mu+\mu^{2} h=2 f .
$$

Recalling that af $=1$ the first equation gives $h=\beta-a \lambda^{2}-a \mu^{2} t$ and the second $2 a \lambda \mu+a \mu^{2} h=2$. Substituting for $h$ in the latter gives

$$
2 a \lambda \mu-a^{2} \mu^{2} \lambda^{2}-a^{2} \mu^{4} t=2 .
$$

The additional demand that $\phi$ is an involution and respects multiplication and comultiplication does not introduce any further relations.

Thus $\theta$ must be of the form $\theta=\lambda 1+\mu x$ and $\phi$ must be of the form $\phi(1)=1$ and $\phi(x)=\beta 1+x$ where $\lambda, \mu, \beta \in U$ satisfy

$$
\mu \beta=\lambda \beta=0 \quad \text { and } \quad 2 a \lambda \mu-a^{2} \mu^{2} \lambda^{2}-a^{2} \mu^{4} t=2,
$$

and there are no further relations necessary. Along with the relation $a f=1$ these are the relations defining $K$ so there is a map $\psi: K \rightarrow R$ as required. Noting that $h=\beta-a \lambda^{2}-a \mu^{2} t$ one easily sees that $U_{\psi} \cong V$.

\section{The complex of cobordisms for a link diagram on a surface}

We consider oriented link diagrams on closed orientable surfaces (as depicted for example in Figure 2) and define an equivalence relation on such diagrams as follows. Two diagrams are said to be stably equivalent if they are related by

(1) surface homeomorphisms

(2) Reidemeister moves

(3) addition or subtraction of handles to the surface (when no part of the diagram is on the handle). 


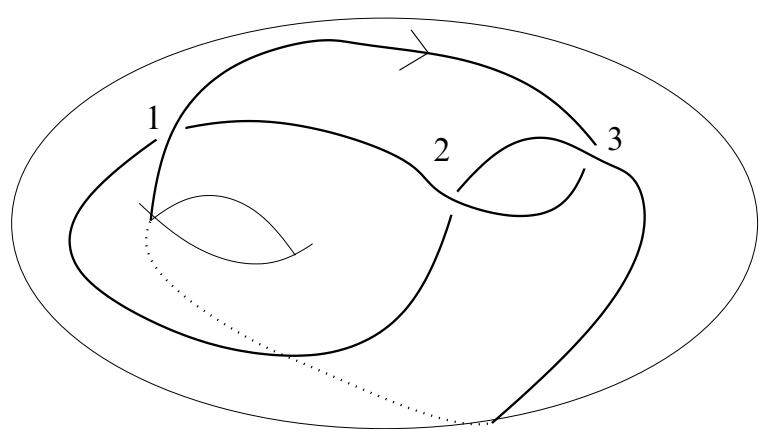

Figure 2: An oriented knot diagram on a torus

Given a link diagram on a closed orientable surface $\Sigma$ we wish to define a complex of cobordisms along the lines of Bar-Natan's complex for link diagrams given in [5].

Recall that for an additive category $\mathcal{C}$ the additive category $\mathcal{M a t}(\mathcal{C})$ is defined as follows. Its objects are finite families $\left\{C_{i}\right\}_{i}$ of objects $C_{i} \in \mathcal{C}$ which for convenience will be denoted $\oplus C_{i}$. A morphism $F: \oplus C_{i} \rightarrow \oplus C_{j}^{\prime}$ is a matrix $F=\left[F_{i}^{j}\right]$ of morphisms $F_{i}^{j}: C_{i} \rightarrow C_{j}^{\prime}$ in $\mathcal{C}$. We will refer to the $F_{i}^{j}$ as the matrix elements of the morphism $F_{j}$. Composition is defined in terms of matrix elements by the rule $[F \circ G]_{i}^{k}=\sum_{j} F_{j}^{k} \circ G_{i}^{j}$ and addition of morphisms given by matrix addition. If $\mathcal{C}$ is not additive then it is made so by allowing formal $\mathbb{Z}$-linear combinations of morphisms.

Define $\mathcal{U C o b}(\Sigma)$ to be the following category. The objects are collections of disjoint closed curves $\Gamma$ in $\Sigma$. A morphism $\Gamma \rightarrow \Gamma^{\prime}$ is a surface embedded in $\Sigma \times I$ whose boundary lies entirely in $\Sigma \times\{0,1\}$ and which agrees with $\Gamma$ on $\Sigma \times\{0\}$ and with $\Gamma^{\prime}$ on $\Sigma \times\{1\}$. Two such morphisms are identified if they are related by a boundary preserving isotopy. Since cobordisms are embedded one can clearly compose them (rescaling the result).

Given an oriented link diagram $D$ on an orientable surface $\Sigma$ we first number the crossings $1, \ldots, n$. We can resolve each crossing in one of two ways as depicted in Figure 3.

A resolution of each of the $n$ crossings of $D$ will be called a smoothing. There are $2^{n}$ such smoothings and each is indexed by a sequence $s$ of $n 0$ 's and 1's, the $i$-th entry informing us whether the $i$-th crossing is a $0-$ or 1 -smoothing. The smoothing itself consists of a closed 1-manifold $\Gamma_{s}$ being a collection of nonintersecting closed curves in $\Sigma$. Note that $\Gamma_{S}$ is an object in the category $\mathcal{U C o b}(\Sigma)$. Smoothings form a poset via

$s<t$ iff all $1-$ smoothings in $s$ are 1 -smoothings in $t$. 


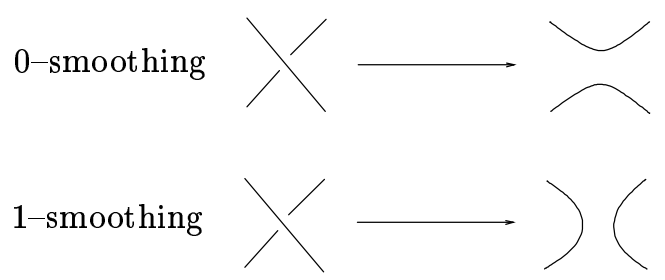

Figure 3

Let $r(s)=$ number of 1 -smoothings in $s$

and $k(s)=$ number of components in $\Gamma_{s}$.

As is familiar the smoothings are arranged on the vertices of a cube with an arrow from $s$ to $t$ if $s<t$ and $r(t)=r(s)+1$. The cube for the knot given in Figure 2 is presented in Figure 4 where the underlying torus has been omitted.

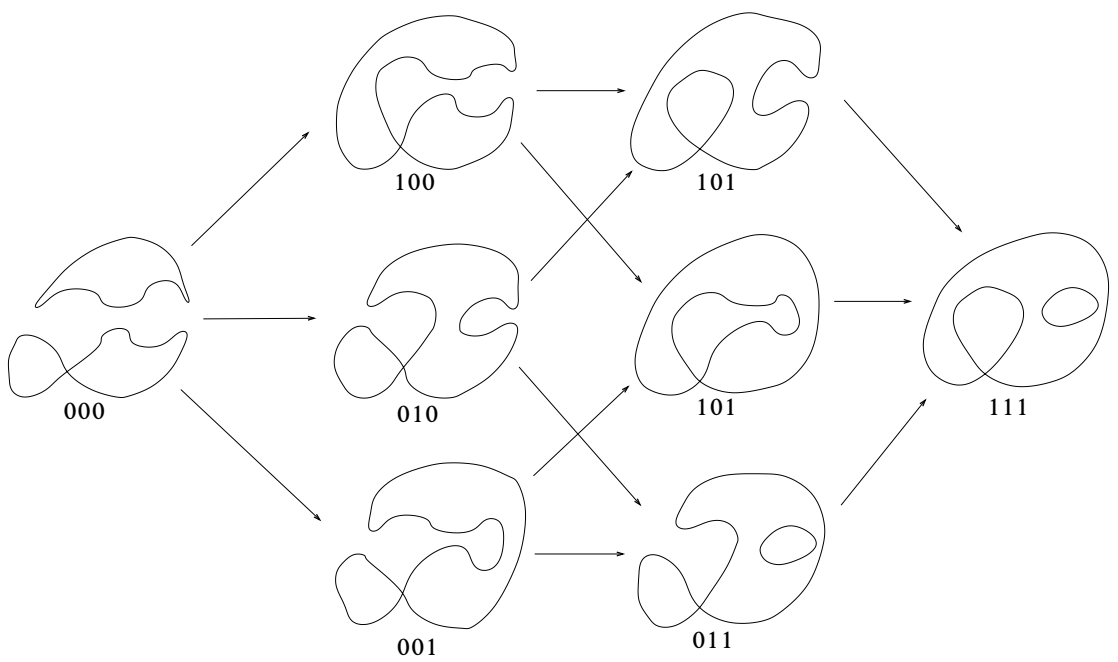

Figure 4: The cube of the diagram in Figure 2

If $s$ and $t$ are smoothings such that $r(t)=r(s)+1$ and $s<t$ then $\Gamma_{t}$ must be identical to $\Gamma_{s}$ outside a small disc in $\Sigma$ around a $1-$ smoothing in $\Gamma_{t}$. We refer to this disc as the changing disc. In this situation we define $\langle s, t\rangle$ to be the number of 1 -smoothings among the first $j-1$ crossings of $t$ where the $j$-th crossing is the one in the changing disc.

For such $s$ and $t$ define $W_{s}^{t} \subset \Sigma \times I$ to be the surface which is $\Gamma_{s}$ on $\Sigma \times\{0\}, \Gamma_{1}$ on $\Sigma \times\{1\}$, a product outside (changing disc) $\times I$ and a saddle in place of the missing 
(changing disc) $\times I$. We regard $W_{s}^{t}$ as a morphism $\Gamma_{s} \rightarrow \Gamma_{t}$ in the category $\mathcal{U C} \operatorname{ob}(\Sigma)$.

Set

$$
[[D]]^{i}=\bigoplus_{s} \Gamma_{s} \in \operatorname{Ob}(\operatorname{Mat}(\mathcal{U C} o b(\Sigma)))
$$

where the sum is over all smoothings $s$ with $r(s)=i+n_{-}$. Here $n_{-}$is the number of negative crossings in $D$.

We now want to define a morphism $d^{i}:[[D]]^{i} \rightarrow[[D]]^{i+1}$. In order to define $d^{i}$ it is enough to define its matrix elements $\left(d^{i}\right)_{s}^{t}: \Gamma_{s} \rightarrow \Gamma_{t}$ where $s$ and $t$ are smoothings such that $r(s)=i+n_{-}$and $r(t)=i+1+n_{-}$. Define these matrix elements by

$$
\left(d^{i}\right)_{s}^{t}= \begin{cases}(-1)^{\langle s, t\rangle} W_{s}^{t} & \text { if } s<t \\ 0 & \text { else. }\end{cases}
$$

Proposition 3.1 Given a diagram $D$, the morphism $d$ defined above satisfies $d^{2}=0$. Thus $\left([[D]]^{*}, d\right)$ is a complex in $\operatorname{Mat}(\mathcal{U C} \operatorname{cob}(\Sigma))$.

Proof Given a square face of the cube

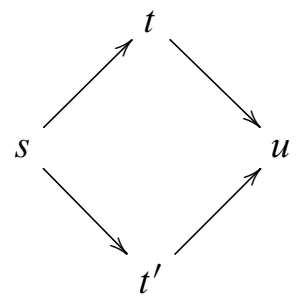

we note that $W_{t}^{u} \circ W_{s}^{t} \cong W_{t^{\prime}}^{u} \circ W_{s}^{t^{\prime}}$ since saddles can be reordered. The signs chosen in the definition of the matrix elements ensure that each square anticommutes.

We continue following [5] by quotienting the category $\mathcal{U C} o b(\Sigma)$ by certain relations. These relations, the $S, T, 4-T u$ (Sphere, Torus and 4-Tube) relations are illustrated below in Figure 5.

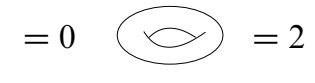

$S$
$T$

Figure 5: Bar-Natan's relations 
Definition 3.2 Let $\mathcal{U C} o b(\Sigma)_{/ r}$ be the category obtained from $\mathcal{U C} o b(\Sigma)$ by quotienting by the equivalence relation generated by relations $S, T, 4-T u$.

Proposition 3.3 Let $D$ be a link diagram on a closed orientable surface $\Sigma$. The homotopy type of the complex $([[D]], d)$ in $\operatorname{Mat}\left(\mathcal{U C o b}(\Sigma)_{/ r}\right)$ is invariant under Reidemeister moves.

Proof In [5] Bar-Natan has proved the analogous statement for planar link diagrams. His proofs remain valid in our setting too.

\section{Link homology}

\subsection{Applying an unoriented TQFT to get link homology}

In this section we will apply a rank-two aspherical unoriented TQFT to the formal complex of cobordisms of the previous section to obtain a complex of $R$-modules. We can then take homology of this complex to obtain a calculable invariant of stable equivalence classes of link diagrams on surfaces.

While geometrically the 4-Tu relation is a genuine relation on cobordisms, algebraically it comes for free for aspherical theories.

Proposition 4.1 Let $(A, \tau)$ be a rank-two aspherical unoriented TQFT. Suppose cobordisms $W_{1}, W_{2}, W_{3}, W_{4}$ are related locally as in the 4-Tu relation above then $\tau\left(W_{1}\right)+\tau\left(W_{2}\right)=\tau\left(W_{3}\right)+\tau\left(W_{4}\right)$.

Proof In order to do computations we need to work with the underlying Frobenius algebra and in order to do that we need to pick identifications $\gamma_{i}: V \rightarrow \Gamma_{i}, i=1,2,3,4$, where the $\Gamma_{i}$ are the four circles appearing $W_{1}$ (and $W_{2}, W_{3}, W_{4}$ ) where the local surgery takes place. (Note that we are assuming that $W_{1}, W_{2}, W_{3}, W_{4}$ are actually the same manifold outside the ball in which the local change takes place.) We need to show:

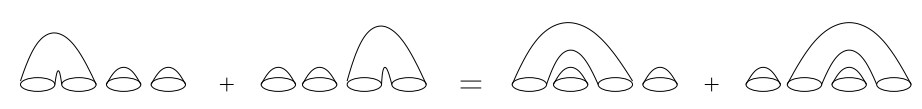

which by using the identifications $\gamma_{i}$ can be converted into the condition on the underlying Frobenius algebra:

$\Sigma a^{\prime} \otimes a^{\prime \prime} \otimes 1 \otimes 1+\Sigma 1 \otimes 1 \otimes a^{\prime} \otimes a^{\prime \prime}=\Sigma a^{\prime} \otimes 1 \otimes a^{\prime \prime} \otimes 1+\Sigma 1 \otimes a^{\prime} \otimes 1 \otimes a^{\prime \prime}$ 
where we write $\Delta(1)=\Sigma a^{\prime} \otimes a^{\prime \prime}$. By Proposition 2.15 it suffices to consider the universal extended Frobenius algebra. Since here $\Delta(1)=f(1 \otimes x+x \otimes 1)-h f 1 \otimes 1$ it is easy to verify that the above equation holds.

Let $(A, \tau)$ be a rank-two aspherical unoriented TQFT. We can now define a functor $\mathcal{U C o b}(\Sigma) \rightarrow \operatorname{Mod}_{R}$ by

$$
\begin{gathered}
\Gamma \mapsto A_{\Gamma}, \\
W \mapsto \tau(W) .
\end{gathered}
$$

This does indeed give a functor since by axiom (4) in Definition 2.1 homeomorphisms preserving the boundary point-wise induce the same map $\tau$ and composition in $\mathcal{U C} \circ b(\Sigma)$ glues along the identity map and so $\tau\left(W^{\prime} \circ W\right)=\tau\left(W^{\prime}\right) \circ \operatorname{Id} \circ \tau(W)$. Note also that by axiom (7) cylinders give the identity homomorphism.

By taking formal direct sums to genuine direct sums this extends to a functor

$$
\mathcal{M a t}(\mathcal{U C o b}(\Sigma)) \rightarrow \operatorname{Mod}_{R}
$$

Since $(A, \tau)$ is rank-two and aspherical, Proposition 4.1 shows that this functor factors through $\operatorname{Mat}\left(\mathcal{U C o b}(\Sigma)_{/ r}\right)$ and hence there is a functor $F_{(A, \tau)}$ on the associated categories of complexes

$$
F_{(A, \tau)}: \operatorname{Kom}\left(\mathcal{M a t}\left(\mathcal{U C} \operatorname{ob}(\Sigma)_{/ r}\right)\right) \rightarrow \mathcal{K} \operatorname{om}\left(\operatorname{Mod}_{R}\right)
$$

Given a link diagram $D$ on a surface set

$$
C_{(A, \tau)}^{*}(D)=F_{(A, \tau)}\left(\left[[D]^{*}\right) .\right.
$$

Proposition 4.2 The homotopy type of the complex $C_{(A, \tau)}^{*}(D)$ is an invariant of stable equivalence classes.

Proof Let $\Sigma \rightarrow \Sigma^{\prime}$ be a homeomorphism of surfaces. This induces homeomorphisms $\Gamma_{s} \rightarrow \Gamma_{s}^{\prime}$ on smoothings and homeomorphisms $W_{s}^{t} \rightarrow\left(W^{\prime}\right)_{s}^{t}$ on the cobordisms defining the complexes. Applying the TQFT gives isomorphisms $A_{\Gamma_{s}} \rightarrow A_{\Gamma_{s}^{\prime}}$ which in turn induce an isomorphism (of graded vector spaces) $C_{(A, \tau)}^{*}(D) \rightarrow C_{(A, \tau)}^{*}(D)^{\prime}$. By axiom (4) in the definition of TQFT we see that this is an isomorphism of complexes.

Showing invariance under Reidemeister moves is the content of Proposition 3.3.

If $\Sigma^{\prime}$ is obtained from $\Sigma$ by the addition or subtraction of handles disjoint from the diagram then clearly there is a canonical identification of smoothings and cobordisms in the complexes $[[D]]^{\prime}$ and $[[D]]$ (defined above using $\Sigma^{\prime}$ and $\Sigma$ ). These identifications lead to an isomorphism of complexes $C_{(A, \tau)}^{*}(D) \rightarrow C_{(A, \tau)}^{*}(D)^{\prime}$. 
Thus if $L$ is a stable equivalence class of diagrams we can now define what we mean by link homology.

Definition 4.3 The link homology for stable equivalence classes based on the TQFT $(A, \tau)$ is defined by

$$
H_{(A, \tau)}^{*}(L)=H\left(C_{(A, \tau)}^{*}(D)\right)
$$

where $D$ is any representative diagram of the class $L$.

Note that if we replace the unoriented TQFT $(A, \tau)$ with an isomorphic one $\left(A^{\prime}, \tau^{\prime}\right)$ then there are isomorphisms $\eta: A_{\Gamma_{s}} \rightarrow A_{\Gamma_{s}}^{\prime}$. As these are natural with respect to cobordisms they induce an isomorphism of complexes $C_{(A, \tau)}^{*}(D) \rightarrow C_{\left(A^{\prime}, \tau^{\prime}\right)}^{*}(D)$. Thus isomorphic TQFTs result in isomorphic link homology groups.

We now present our main result.

Theorem 4.4 Let $R$ be a commutative ring with unit. Let $\lambda, \mu, \beta, a, t \in R$ such that $a$ is invertible and the following relations are satisfied:

$$
\begin{gathered}
\mu \beta=\lambda \beta=0, \\
2 a \lambda \mu-a^{2} \mu^{2} \lambda^{2}-a^{2} \mu^{4} t=2 .
\end{gathered}
$$

Then the 5-tuple ( $\lambda, \mu, \beta, a, t)$ defines (the isomorphism class of) a link homology theory for stable equivalence classes of diagrams on oriented surfaces.

Proof The obvious map $\psi: K \rightarrow R$ defines a rank-two extended Frobenius algebra $(V, \theta, \phi)$ satisfying $\epsilon(i(1))=0$. This in turn defines a rank-two aspherical $1+1-$ dimensional unoriented TQFT. Such a theory defines a link homology for stable equivalence classes as described above.

Corollary 4.5 If $R$ is an integral domain then each triple $(a, \lambda, \mu)$ with $a$ and $\mu$ invertible defines a link homology.

\subsection{Link homologies over $\mathbb{Q}$}

Clearly there are solutions to equations (1) and (2) over $\mathbb{Q}$. The question we now address is whether or not any of these extend a theory isomorphic to Khovanov's original link homology. Given $h, t \in \mathbb{Q}$ then there is a link homology defined by the Frobenius algebra $\mathbb{Q}\{1, x\}$ with $i(1)=1, \epsilon(1)=0, \epsilon(x)=1$ and $x^{2}=h x+t 1$. Recall from [11] that such a theory is isomorphic to Khovanov's original theory if and only if 
$h^{2}+4 t=0$. Noting that over $\mathbb{Q}$ we must have $\beta=0$ and $\mu \neq 0$ we can express $h$ and $t$ in terms of $\lambda$ and $\mu$ as

$$
h=2 \mu^{-2}-2 \lambda \mu^{-1} \text { and } \quad t=-\mu^{-2} \lambda^{2}-2 \mu^{-4}+2 \lambda \mu^{-3} .
$$

A computation now shows

$$
h^{2}+4 t=-4 \mu^{-4} \text {. }
$$

Thus we have $h^{2}+4 t \neq 0$ resulting in the disappointing conclusion that Khovanov's original theory does not extend (at least using the methods of this paper) to stable equivalence classes. This conclusion remains valid over fields of characteristic not equal to two.

Over $\mathbb{Q}$ the resulting theories are all singly graded. For each of these the Euler characteristic, $\chi$, is the unnormalised Jones polynomial evaluated at $q=1$. To see this recall that the Euler characteristic of the homology of a complex is the same as the Euler characteristic of the complex itself and so

$$
\begin{aligned}
\chi=\sum_{i}(-1)^{i} \operatorname{dim}\left(H^{i}(D)\right) & =\sum_{i}(-1)^{i} \operatorname{dim}\left(F_{(A, \tau)}\left([[D]]^{i}\right)\right) \\
& =\sum_{i}(-1)^{i} \sum_{\substack{s \in \text { smoothings } \\
r(s)=i+n_{-}}} \operatorname{dim}\left(A_{\Gamma_{s}}\right)=\sum(-1)^{i} \sum_{\substack{s \in \text { smoothings } \\
r(s)=i+n_{-}}} 2^{k(s)}
\end{aligned}
$$

where $k(s)$ is the number of components in $\Gamma_{s}$. Comparing with the definition of the Jones polynomial we see that the right-hand side is the unnormalised Jones polynomial evaluated at 1 .

\subsection{Link homologies over $\mathbb{F}_{2}$}

Working over $\mathbb{F}_{2}$ one has more success than over the rationals. One has $a=1$ and the remaining equations of the main theorem become

$$
\mu \beta=\lambda \beta=0 \quad \text { and } \quad \mu^{2} \lambda^{2}+\mu^{4} t=0 .
$$

There are eight possibilities as tabulated below. 


\begin{tabular}{|c|c|c|c|c|c|c|}
\hline$\lambda$ & $\mu$ & $t$ & $\beta$ & $h$ & $\theta$ & $\phi(x)$ \\
\hline 0 & 0 & 0 & 0 & 0 & 0 & $x$ \\
0 & 0 & 0 & 1 & 1 & 0 & $1+x$ \\
1 & 0 & 0 & 0 & 1 & 1 & $x$ \\
0 & 0 & 1 & 0 & 0 & 0 & $x$ \\
0 & 0 & 1 & 1 & 1 & 0 & $1+x$ \\
1 & 0 & 1 & 0 & 1 & 1 & $x$ \\
0 & 1 & 0 & 0 & 0 & $x$ & $x$ \\
1 & 1 & 1 & 0 & 0 & $1+x$ & $x$ \\
\hline
\end{tabular}

Rows 1, 4, 7 and 8 have isomorphic underlying Frobenius algebras and on classical links give theories that are isomorphic to Khovanov's original theory over $\mathbb{F}_{2}$. As extended Frobenius algebras there are two isomorphism classes among these with row 1 isomorphic to row 4 and row 7 isomorphic to row 8 . The theory in the first row gives a bigraded theory and was investigated by Manturov [12]. He observes that the graded Euler characteristic (which can be computed from the graded Euler characteristic of the chain complex) is the unnormalised Jones polynomial of the link.

The theory in row 3 can be made into a bigraded theory by taking $R=\mathbb{F}_{2}[\lambda]$ with $\lambda$ in degree -1 . The Frobenius algebra has multiplication

$$
11=1, \quad 1 x=x 1=x \quad \text { and } \quad x x=\lambda^{2} x
$$

and comultiplication

$$
\begin{gathered}
\Delta(1)=1 \otimes x+x \otimes 1+\lambda^{2} 1 \otimes 1, \\
\Delta(x)=x \otimes x .
\end{gathered}
$$

The counit is $\epsilon(1)=0, \epsilon(x)=1$. This is isomorphic to Bar-Natan's graded characteristic two theory. For the extended structure we have $\theta=\lambda 1$ and $\phi=\mathrm{Id}$. This theory is related to Manturov's theory: one can filter the chain complex by powers of $\lambda$ to produce a spectral sequence (similar to that in [14]) whose $E_{2}$-page is Manturov's theory and which converges to the bigraded theory.

It is interesting to note that this extended Frobenius algebra has an interpretation in terms of the equivariant cohomology of $S^{2}$ along the lines of [10]. Let $G=\mathbb{Z} / 2$ act on $S^{2}$ by a rotation through $\pi$ about a fixed axis. In this case we have

$$
H_{G}^{*}\left(\mathrm{pt} ; \mathbb{F}_{2}\right)=H^{*}\left(\mathbb{R} P^{\infty} ; \mathbb{F}_{2}\right)=\mathbb{F}_{2}[\lambda]
$$

and

$$
H_{G}^{*}\left(S^{2} ; \mathbb{F}_{2}\right)=\mathbb{F}_{2}[\lambda, x] /\left\langle x^{2}=\lambda^{2} x\right\rangle=\mathbb{F}_{2}[\lambda][x] /\left\langle x^{2}=\lambda^{2} x\right\rangle .
$$


We see that this is the algebra of the bigraded link homology above and the element $\theta$ is the image of the generator of $H_{G}^{*}\left(\mathrm{pt} ; \mathbb{F}_{2}\right)$ under the homomorphism induced from $S^{2} \rightarrow \mathrm{pt}$.

Bigraded Bar-Natan theory is also extended by the theory in row 2 . Here we work over $R=\mathbb{F}_{2}[\beta]$ with $\beta$ in degree -2 . In this case $\theta=0$ and $\phi(x)=\beta 1+x$. Note that in this case we have a nontrivial involution $\phi$.

\subsection{Acknowledgments}

We thank D Bar-Natan and G Naot for many helpful comments. The second author was supported by the European Commission through a Marie Curie fellowship and thanks the Institut de Recherche Mathématique Avancée in Strasbourg for their hospitality.

\section{References}

[1] A Alexeevski, S Natanzon, Noncommutative two-dimensional field theories and Hurwitz numbers for real algebraic curves arXiv:math.GT/0202164

[2] M M Asaeda, J H Przytycki, A S Sikora, Categorification of the Kauffman bracket skein module of I -bundles over surfaces, Algebr. Geom. Topol. 4 (2004) 1177-1210 MR2113902

[3] M Atiyah, Topological quantum field theories, Inst. Hautes Études Sci. Publ. Math. (1988) 175-186 (1989) MR1001453

[4] D Bar-Natan, On Khovanov's categorification of the Jones polynomial, Algebr. Geom. Topol. 2 (2002) 337-370 MR1917056

[5] D Bar-Natan, Khovanov's homology for tangles and cobordisms, Geom. Topol. 9 (2005) 1443-1499 MR2174270

[6] R Fenn, L Kauffman, V Manturov, Virtual Knot Theory - Unsolved Problems arXiv:math.GT/0405428

[7] M Jacobsson, An invariant of link cobordisms from Khovanov homology, Algebr. Geom. Topol. 4 (2004) 1211-1251 MR2113903

[8] L Kauffman, V Manturov, Virtual Knots and Links arXiv:math.GT/0502014

[9] M Khovanov, A categorification of the Jones polynomial, Duke Math. J. 101 (2000) 359-426 MR1740682

[10] M Khovanov, Link homology and Frobenius extensions, Fund. Math. 190 (2006) 179190

[11] M Mackaay, P Turner, P Vaz, A remark on Rasmussen's invariant of knots arXiv: math.GT/0509692 
[12] V Manturov, The Khovanov complex for virtual links arXiv:math.GT/0501317

[13] V G Turaev, Quantum invariants of knots and 3-manifolds, de Gruyter Studies in Mathematics 18, Walter de Gruyter \& Co., Berlin (1994) MR1292673

[14] P Turner, Calculating Bar-Natan's characteristic two Khovanov homology arXiv: math.GT/0411225

Institut de Recherche Mathématique Avancée

7 rue René Descartes, 67000 Strasbourg, France

School of Mathematical and Computer Sciences, Heriot-Watt University

Edinburgh EH14 4AS, Scotland

turaev@math.u-strasbg.fr, paul@ma.hw.ac.uk

Received: 1 September 2005 Revised: 16 January 2006 